\title{
Nanometer Scale Magnetic Linear Dichroism by EELS
}

\author{
Y. Ito***, M. van Veenendaal***, R.E. Cook**, N. Menon***, B.D. Armstrong*, D.J. Miller** \\ *Department of Physics, Northern Illinois University, DeKalb, IL 60115 \\ **Materials Science Division, Argonne National Laboratory, 9700 S. Cass Ave. Argonne, IL 60439 \\ ***Gatan Inc.
}

Since the advent of the spin-based electronics, tremendous efforts have been focused on fabrication and characterization of nanomagnetic structures. Since the spin transport occurs through the bulk of multi-layered nanostructures, probes directly sensitive to the magnetic anisotropy of the bulk and its interfaces are of great interest. So far, intense research activities to probe the magnetic anisotropy have been centered on polarized synchrotron X-ray spectroscopy techniques. However, the spatial resolution of these techniques is currently limited to $\sim 50 \mathrm{~nm}$. Therefore, there is an urgent need for a technique capable of probing the magnetic anisotropy on length scale less than a few nanometers.

Yuan and Menon showed that it is possible to detect nanometer scale magnetic anisotropy, or magnetic linear dichroism (MLD), using STEM-based momentum-resolved EELS [1,2]. They showed that the difference of two spectra is proportional to the magnetic linear dichroism. However, there has been neither further confirmation nor development on this potentially powerful method.

Here, we present an investigation of the MLD in a model sample, $\alpha-\mathrm{Fe}_{2} \mathrm{O}_{3}$ (hematite) [3], using the momentum-resolved EELS with a sub-nanometer electron probe in a $200 \mathrm{kV}$ Schottky field emission TEM/STEM (Philips FEI Tecnai F20). The energy resolution was $<0.9 \mathrm{eV}$ (FWHM of zero loss peak). $\alpha-\mathrm{Fe}_{2} \mathrm{O}_{3}$ has antiferromagnetic ground state with spins on specific neighboring planes oriented in opposite directions. At room temperature, its magnetic moments are perpendicular to the c-axis. $\mathrm{Fe}_{2} \mathrm{O}_{3}$ nanoparticles may have application to storage, gas sensing, etc.

MLD can be extracted by taking the difference of two spectra acquired from an oriented single crystal with different convergence angles $(\alpha)$ with the EELS collection angle $(\beta)$ fixed, hence changing the ratio of parallel to perpendicular components of the scattering vector (FIG. 1). FIG. 2 shows the experimental $\mathrm{Fe} \mathrm{L}_{23}$ EEL spectra acquired with different convergence angles (18.5 mrad and $8.7 \mathrm{mrad}$ ) and their difference spectrum. The incident beam was parallel to the c-axis of the oriented microcrystal. The difference spectrum clearly shows negative peaks. This agrees with the calculated MLD spectrum based on the atomic multiplet calculation for the octahedrally coordinated $\mathrm{Fe}^{3+}$ ion in $\alpha-\mathrm{Fe}_{2} \mathrm{O}_{3}$ (Fig. 3 and 4), although the experimental spectra do not reproduce fine peaks due to insufficient energy resolution. The present result is also consistent with the result obtained from the synchrotron X-ray MLD experiment on a single crystal $\alpha-\mathrm{Fe}_{2} \mathrm{O}_{3}$ [4].

One important factor was noted in these experiments. Use of a longer exposure time may produce a positive difference spectrum due to beam damage, i.e., reduction of $\mathrm{Fe}^{3+}$ to $\mathrm{Fe}^{2+}$. Therefore, these measurements require careful control, especially exposure time. 


\section{References}

[1] J. Yuan, N.K. Menon, J. Appl. Phys. 81, (1997) 5087.

[2] N.K. Menon, J. Yuan, Ultramicroscopy 78, (1999) 185.

[3] C.G. Shull et al., Phys. Rev. 83 (1951) 333.

[4] P. Kuiper et al., Phys. Rev. Lett. 70 (1993), 1549.

[5] This work is supported by the State of Illinois under HECA, NIU URA program, and work at Argonne, carried out in the Electron Microscopy Center, is supported by the U.S. Department of Energy, Basic Energy Sciences-Materials Sciences, under Contract \#W-31-109-ENG-38.

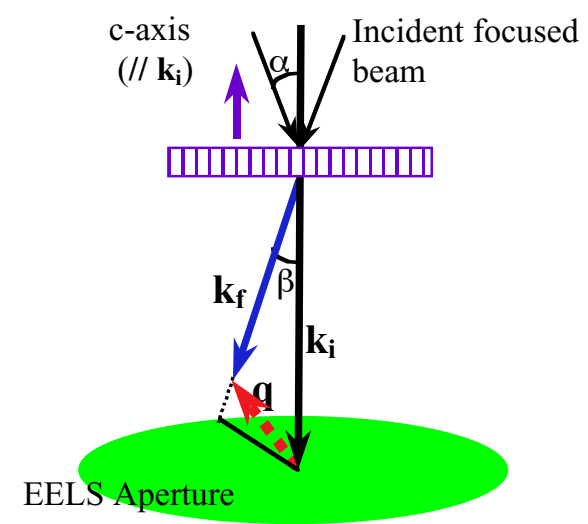

FIG. 1. Schematic of technique for Magnetic Linear Dichroism.

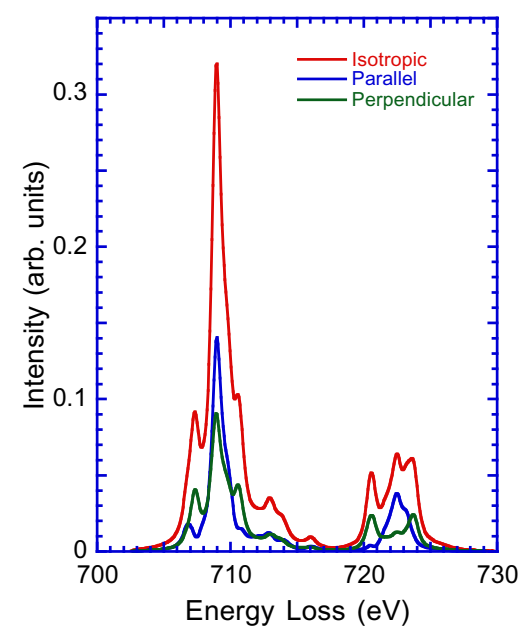

FIG. 3. Theoretical Fe $\mathrm{e}^{3+}$ spectrum in $\alpha-$ $\mathrm{Fe}_{2} \mathrm{O}_{3}$ and its components. Spin orientation is perpendicular to c-axis. Parallel component: blue; Perpendicular component: green; Isotropic spectrum: red.

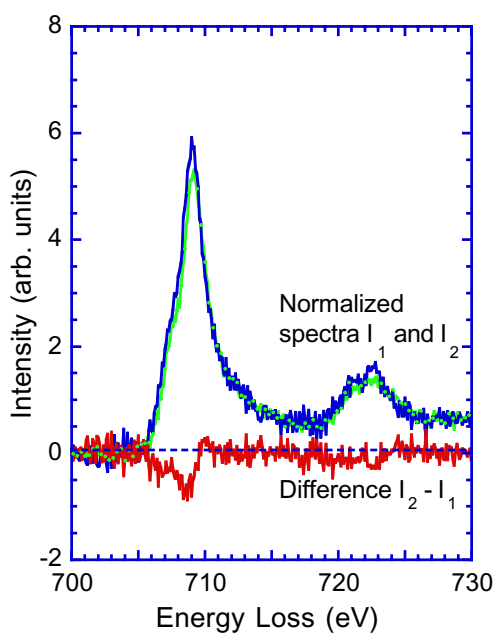

FIG. 2. Experimental $\mathrm{Fe}_{23}$ and difference spectra of $\alpha-\mathrm{Fe}_{2} \mathrm{O}_{3}$ (001) orientation. Condenser apertures: $70 \mu \mathrm{m}$ (blue), $150 \mu \mathrm{m}$ (green). Difference spectrum (red). Exposure: $4.5 \mathrm{sec} / \mathrm{spectrum}$.

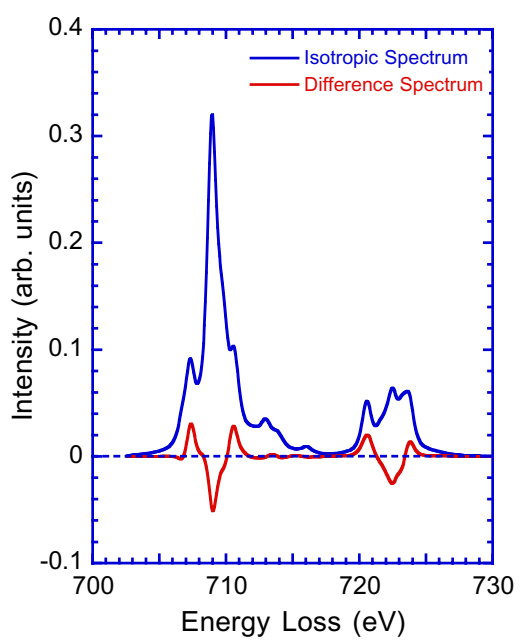

FIG. 4. Theoretical Fe $\mathrm{L}_{23}$ difference spectrum (MLD). The negative peaks at 709 and $722 \mathrm{eV}$ of the difference spectrum indicate that the spin orientation is perpendicular to c-axis. 